\title{
Corrigendum
}

Saba Samad Memon, Kunal Thakkar, Virendra Patil, Swati Jadhav, Anurag R. Lila, Gwendolyn Fernandes, Tushar R. Bandgar* and Nalini S. Shah

\section{Corrigendum to: Primary pigmented nodular adrenocortical disease (PPNAD): single centre experience}

https://doi.org/10.1515/jpem-2019-8888

Corrigendum to: Saba Samad Memon, Kunal Thakkar, Virendra Patil, Swati Jadhav, Anurag R. Lila, Gwendolyn Fernandes, Tushar R. Bandgar, and Nalini S. Shah. 2019. Primary pigmented nodular adrenocortical disease (PPNAD): single centre experience. Journal of Pediatric Endocrinology and Metabolism. Volume 32, Issue 4, pages 391-397. (DOI: https://doi.org/10.1515/jpem-2018-0413):

In the previously published article the acknowledgment and the research funding were incomplete. They should read as follows:

Acknowledgements: We acknowledge Dr. Constantine A. Stratakis and his team from the Section on
Endocrinology and Genetics, Program on Developmental Endocrinology and Genetics, Eunice Kennedy Shriver National Institute of Child Health and Human Development (NICHD), National Institutes of Health (NIH), Bethesda, Maryland, USA for enrolling our patients under the protocol 95-CH-0059 and providing reports of genetic analyses of our patients and for the constant support and care to the families of patients with PPNAD provided by them.

Research funding: The study under protocol 95-CH-0059 and thus the genetic testing performed was supported by the Intramural Research Program, Eunice Kennedy Shriver National Institute of Child Health and Human Development (NICHD), National Institutes of Health.

*Corresponding author: Dr. Tushar R. Bandgar, Professor, Department of Endocrinology and Metabolism, Seth G S Medical College and KEM Hospital, Parel, Mumbai 400012, Maharashtra, India, Phone: 09820025037, E-mail: drtusharb@gmail.com Saba Samad Memon, Kunal Thakkar, Virendra Patil, Swati Jadhav, Anurag R. Lila and Nalini S. Shah: Department of Endocrinology and Metabolism, Seth G S Medical College and KEM Hospital, Parel, Mumbai 400012, Maharashtra, India

Gwendolyn Fernandes: Department of Pathology, Seth G S Medical College and KEM Hospital, Parel, Mumbai, Maharashtra, India 